\title{
Effect of doping concentration on absorbance, structural, and magnetic properties of cobalt-doped $\mathrm{ZnO}$ nano-crystallites
}

\author{
Muhammad Ahsan Shafique ${ }^{1}$, Saqlain A Shah ${ }^{2 *}$, Muhammad Nafees ${ }^{1}$, Khalid Rasheed ${ }^{1,3}$ and Riaz Ahmad ${ }^{1}$
}

\begin{abstract}
Controlled conduction of magnetic spins is desired for data processing in modern spintronic devices. Transition metal-doped $\mathrm{ZnO}$ is a potential candidate for this purpose. We studied the effects of cobalt doping on structural, absorbance, and magnetic properties of $\mathrm{ZnO}$ nano-particles. Different compositions $\left(\mathrm{Zn}_{0.99} \mathrm{Co}_{0.1} \mathrm{O}, \mathrm{Zn}_{0.97} \mathrm{CO}_{0.3} \mathrm{O}\right.$, and $\mathrm{Zn}_{0.95} \mathrm{CO}_{0.5} \mathrm{O}$ ) of cobalt-doped $\mathrm{ZnO}$ were fabricated using metallic chlorides by co-precipitation method. XRD revealed standard $\mathrm{ZnO}$ wurtzite crystal structure without lattice distortion due to impurities but showed presence of additional phases at higher doping ratios. Fourier transformed infrared spectroscopy also confirmed the standard $\mathrm{ZnO}$ profiles at lower doping ratios but additional phases at higher doping. Vibrating sample magnetometer showed soft ferromagnetic behavior for low impurity samples and harder ferromagnetic behavior for higher doping at room temperature. A simultaneous differential scanning calorimetry/thermo gravimetric analysis was performed to study the phase variations during crystallization.
\end{abstract}

Keywords: Cobalt-doped ZnO, DMS, FTIR, VSM, Crystal growth, Metallic chlorides

\section{Background}

$\mathrm{ZnO}$ is not new for researchers. Its fundamental structural properties were studied in 1935 for the first time by Bunn [1], and then detailed optical studies were carried out by Damen et al. [2] and Decremps et al. [3] using Raman spectroscopy.

Initially, $\mathrm{ZnO}$ got the attention of researchers because of its potential applications in laser due to large exciton binding energy $(60 \mathrm{mev})$ and wide band gap about $3.3 \mathrm{ev}$ [4]. Now, $\mathrm{ZnO}$ is being studied because of its promising application for spintronics and optoelectronics.

Spintronics requires controlled conduction and high degree of spin polarization. $\mathrm{ZnO}$ is a semiconductor in nature and has very favorable structure carrier-induced ferromagnetism. After the theoretical study of Dietl et al. in which they observed room temperature ferromagnetism in Mn-doped $\mathrm{ZnO}$ [5], the researchers started a comprehensive study of $\mathrm{Mn}$ and other transition metaldoped $\mathrm{ZnO}$. The electronic structure of doped metal is

\footnotetext{
* Correspondence: saqlain007pk@hotmail.com

${ }^{2}$ Department of Physics, Forman Christian College (University), Lahore 54000, Pakistan

Full list of author information is available at the end of the article
}

strongly affected by the electronic structure of host lattice. There is a strong hybridization of 3D-host energy levels and 3D-3D Coulomb interactions [4]. Initially, some researchers reported absence of ferromagnetism at room temperature. Simultaneously, some of them reported room temperature ferromagnetism but in the materials fabricated at low temperatures [6,7]. In our work, Co-doped $\mathrm{ZnO}$ nano-particles $\left(\mathrm{Zn}_{1}-{ }_{x} \mathrm{Co}_{x} \mathrm{O}\right.$, $x=1,3$, and 7) were successfully fabricated using chemical co-precipitation method to study the effect of doping concentration on structural, absorbance, thermal, and magnetic properties.

\section{Methods}

\section{Experimental procedure}

Pure chemicals $(99.9 \%)$ cobalt chloride $\left(\mathrm{Co}(\mathrm{Cl})_{2}\right)$, zinc chloride $\left(\mathrm{Zn}(\mathrm{Cl})_{2}\right), \mathrm{NaOH}$, and absolute ethanol of a reliable brand were used for this study. $\mathrm{Zn}(\mathrm{Cl})_{2}$ and $\mathrm{Co}(\mathrm{Cl})_{2}$ were taken according to the calculated stoichiometric ratio in a beaker containing absolute ethanol and were stirred for $2 \mathrm{~h}$. $\mathrm{NaOH}$ mixed in ethanol was added to the chloride solution dropwise. The solution was then stirred at room temperature for $2 \mathrm{~h}$. After $2 \mathrm{~h}$, the precipitates were collected from the solution using centrifuge. 
The precipitates were washed several times using absolute ethanol and distilled water. Then, the precipitates were oven dried for $15 \mathrm{~h}$. The product obtained at this stage was zinc and cobalt hydroxide powder.

Finally, the thermal decomposition of the zinc and cobalt hydroxide was used to obtain $\mathrm{Zn}_{x} \mathrm{Co}_{1-}{ }_{-} \mathrm{O}$ nanocrystals. For this purpose, we placed them at temperature $200^{\circ} \mathrm{C}$ for $6 \mathrm{~h}$ to obtain different sizes of nano-crystals. The chemical reaction is

$$
x \mathrm{Zn}(\mathrm{OH})_{2}+(1-x) \mathrm{Co}(\mathrm{OH})_{2} \rightarrow \mathrm{Zn}_{x} \mathrm{Co}_{1-x} \mathrm{O}+\mathrm{H}_{2} \mathrm{O}
$$

The overall chemical reaction is revealed by given chemical equation:

$$
\begin{gathered}
x \mathrm{Zn}(\mathrm{Cl})_{2}+1-x \mathrm{Co}(\mathrm{Cl})_{2}+\mathrm{NaOH} \\
\downarrow \text { Stirring } \\
X \mathrm{Zn}(\mathrm{OH})_{2}+1-x \mathrm{Co}(\mathrm{OH})_{2} \\
\downarrow 200^{\circ} \mathrm{C} \\
\mathrm{Zn}_{x} \mathrm{Co}_{1-x} \mathrm{O}+\mathrm{H}_{2} \mathrm{O}
\end{gathered}
$$

\section{Characterization}

The X'Pert PRO MPD system of PANalytical Ltd. (Almelo, The Netherlands) was used for X-ray diffraction study. A simultaneous differential scanning calorimetry/ thermo gravimetric analysis (DSC/TGA) system of SDT Q600 (TA Instruments, DE, USA) was used for thermogravimetric analysis. Lake Shore VSM 7407 (Lake Shore Cryotonics, Inc., OH, USA) was used for hysteresis studies. FTIR Bruker Tensor 27 (Bruker Daltonik GmbH, Bremen, Germany) was used to study absorbance properties.

\section{Results and discussions}

XRD graphs of doped $\mathrm{ZnO}$ nano-particles (shown in Figure 1) representing the standard picture of pure $\mathrm{ZnO}$

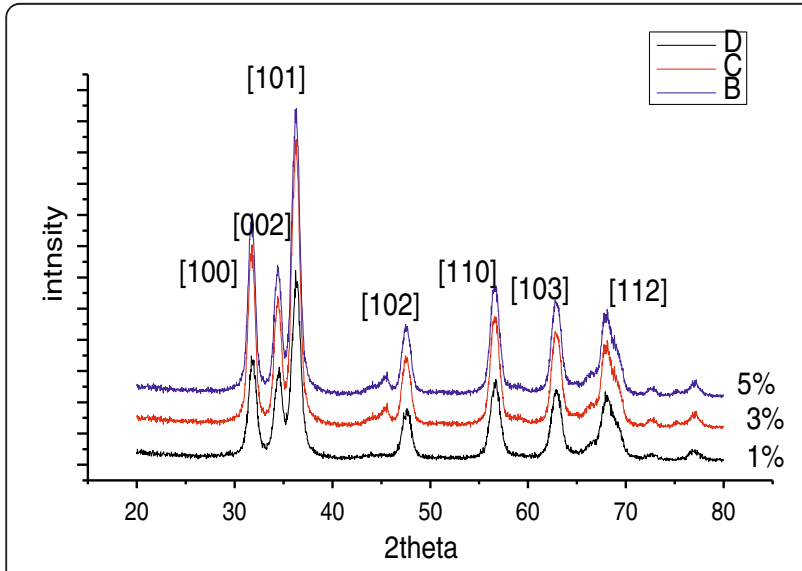

Figure 1 XRD patterns of the three samples $1 \%$, 3\%, and $5 \%$ cobalt-doped $\mathrm{ZnO}$. even at higher doping concentrations show successful substitution of cobalt atoms with zinc atoms [8-10]. But the profiles of higher doping concentrations contain some additional peaks at $2 \theta=47^{\circ}$ which is a diffraction from the cobalt-crystal system. Although these peaks are very feeble, they exist and represent agglomeration of cobalt atoms at higher doping ratios. Distortion or other type of degradation (like shift of peaks and decrease of crystallinity) was not observed in doped samples as claimed by researchers working with $\mathrm{Fe}$ and $\mathrm{Mn}$ as dopants [11,12]. The crystallite size calculated using Scherer's formula was found to be 14.61, 14.9, and $15.27 \mathrm{~nm}$ for $1 \%, 3 \%$, and5\% (impurity vs. crystallite size graphically shown in Figure 2) cobalt-doped $\mathrm{ZnO}$, respectively. A simultaneous DSC/TGA study of precursor was carried out during the fabrication of $\mathrm{ZnO}$. DSC/ TGA SDT Q600 measures amount and rate of change of mass as a function of temperature or time. Our DSC/ TGA instrument is capable of performing both DSC and TGA simultaneously. DSC/TGA analysis was performed to study the change of phases during crystallization. The mixture of $\mathrm{Zn}(\mathrm{OH})_{2}$ and $\mathrm{Co}(\mathrm{OH})_{2}$ was taken before annealing and was put into the furnace of DSC in an inert environment. The simultaneous DSC/TGA graph is shown in Figure 3. The following results were deduced from the obtained data: Since the samples were fabricated in ethanol environment and then washed with distilled water and ethanol several times, the materials contain traces of ethanol. The first peak was observed at $57.45^{\circ} \mathrm{C}$ which represents the mass loss due to evaporation of ethanol. This behavior can be observed from both heat flow and mass loss graphs. The second downward peak in heat flow graph and further decrease of mass was observed at $178.54^{\circ} \mathrm{C}$ and $190.64^{\circ} \mathrm{C}$ in weight curve, which represent decompositions of $\mathrm{Zn}(\mathrm{OH})_{2}$ and $\mathrm{Co}(\mathrm{OH})_{2}$, respectively. Both the materials liberate water molecules. Further, 10\% mass loss was observed at

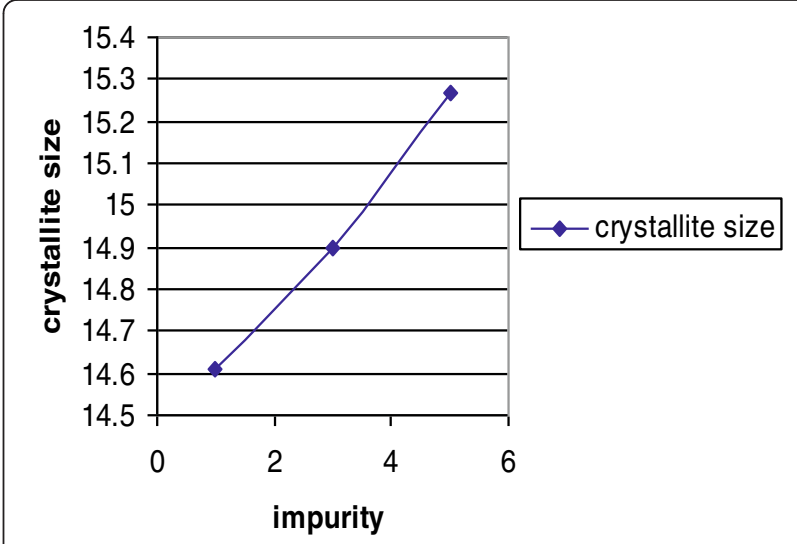

Figure 2 Impurity vs. crystallite size graph. 

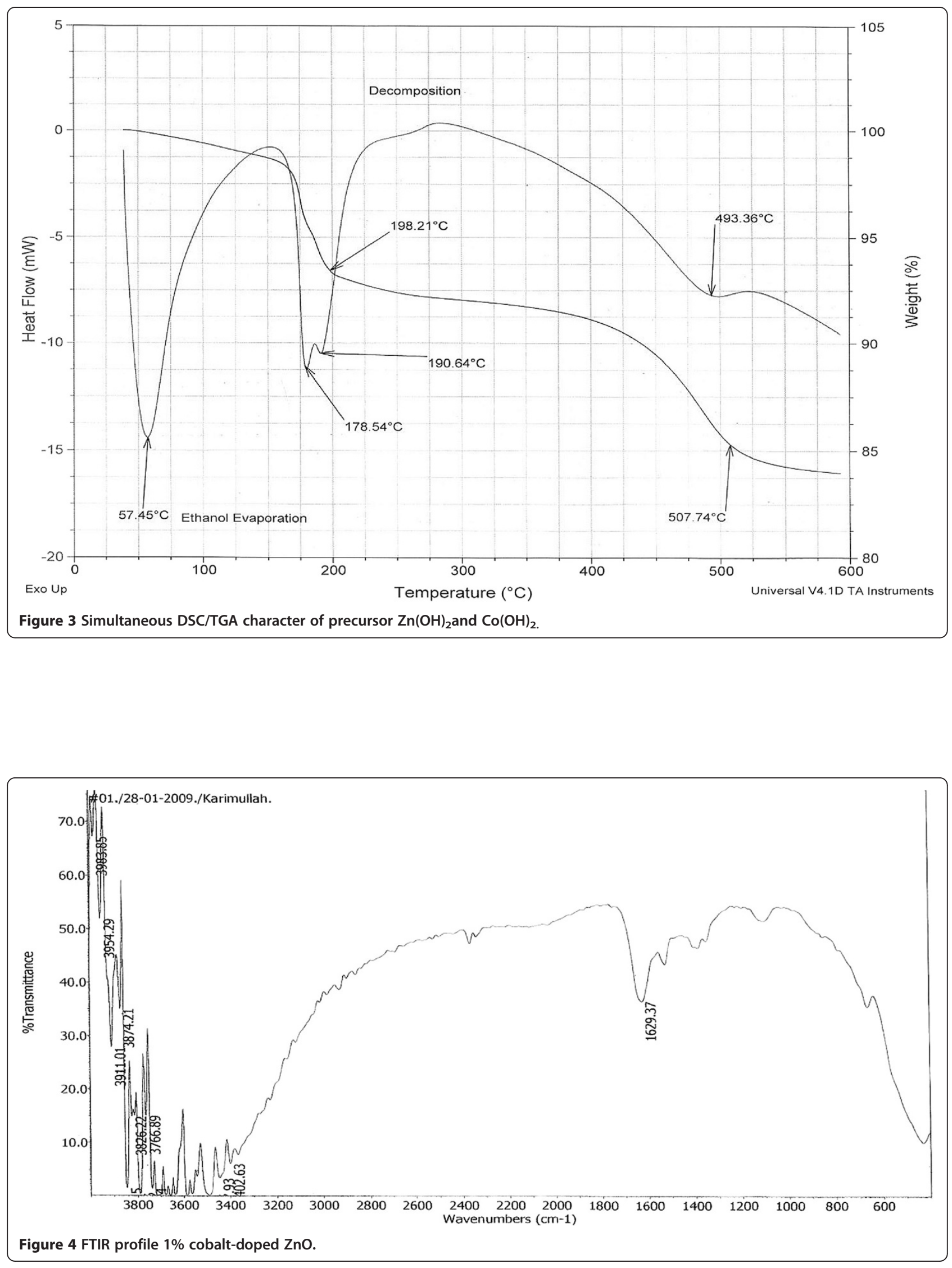

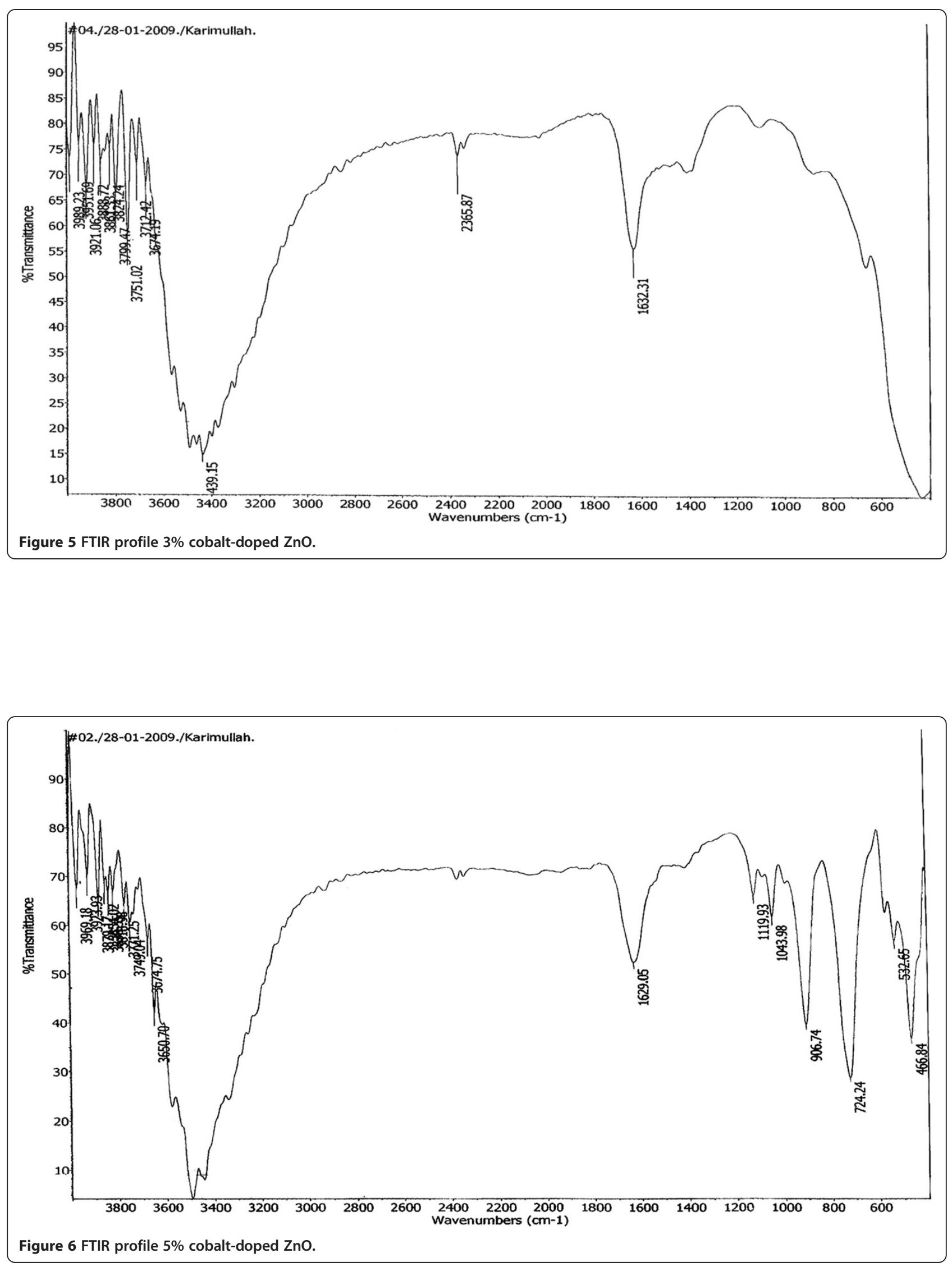


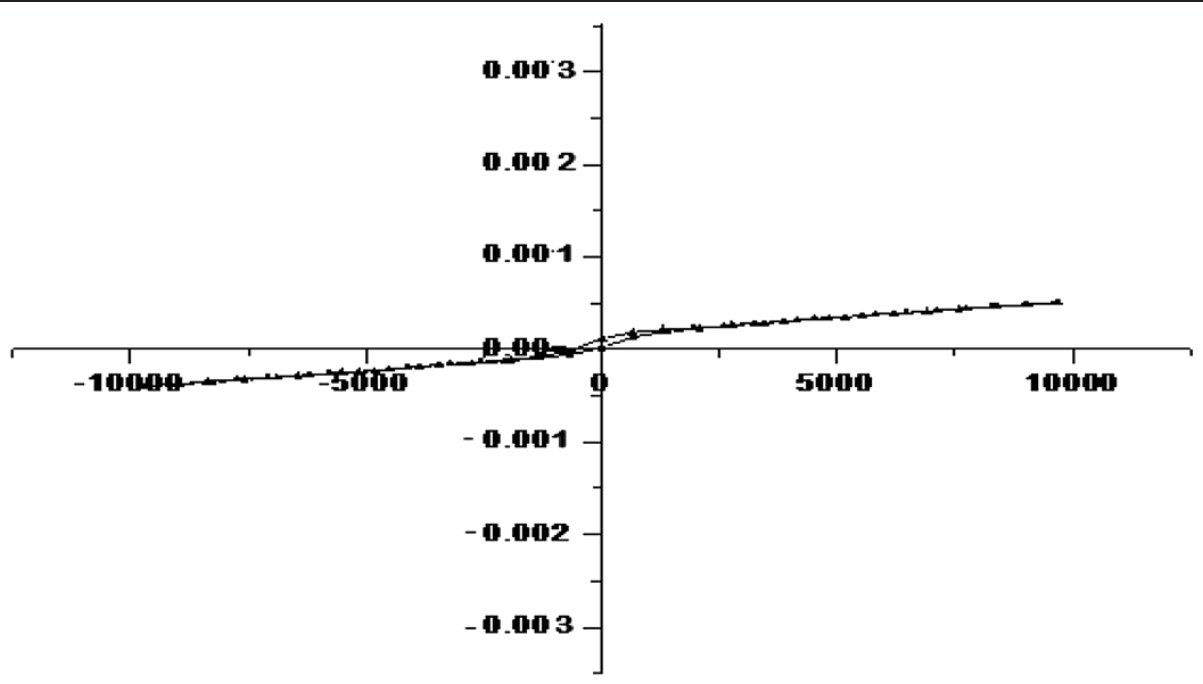

Figure 7 VSM profile of magnetic moment (emu) versus applied field (G) curve of $1 \%$ cobalt-doped ZnO.

approximately $500^{\circ} \mathrm{C}$ due to the evaporation of water of crystallization. These results were in good agreement with the literature [13]. The crystallization occurs during the removal of water molecules. So, heat loss during crystallization could not be observed from the heat graph because this heat is absorbed in the material to liberate water of crystallization. We can observe weight loss at $500^{\circ} \mathrm{C}$ and an endothermic peak of smaller intensity.

Fourier transformed infrared spectroscopy (FTIR), presented in Figures 4, 5, and 6, was performed to study the absorbance properties of fabricated samples and hence to deduce the nature of bonds present in the fabricated samples. In the given profile on wave number axis, the absorbance peaks from wave number 3,400 to $3,900 \mathrm{~cm}^{-1}$ are the peaks representing the hydroxyl $(-\mathrm{OH})$ groups, which show incomplete removal of organic solvent. The next absorbance peak at wave number approximately $1,630 \mathrm{~cm}^{-1}$ is due to the absorbance of organic group carbon (carbonyl carbon $-\mathrm{C}=\mathrm{O}$ ). The absorbance at wave number approximately $466 \mathrm{~cm}^{-1}$ is due to the typical bond between zinc and oxygen ( $\mathrm{Zn}-\mathrm{O}$ ) [14-16]. The peaks from 700 to 900 $\mathrm{cm}^{-1}$ are attributed to the bond between cobalt and oxygen (Co-O) [17]. From the obtained FTIR profiles, we can observe the typical $\mathrm{ZnO}$ peaks and presence of organic contents in $1 \%$ and $3 \%$ doped samples, but in $5 \%$ cobaltdoped $\mathrm{ZnO}$ sample, we can observe additional peaks in wave number region 600 to $1,200 \mathrm{~cm}^{-1}$ which is the region of $\mathrm{ZnO}$ and $\mathrm{Co}-\mathrm{O}$ bonding which again represents the presence of additional phases at higher concentration of doping.

We have done VSM analysis to observe magnetic characteristics of our fabricated samples to study room temperature ferromagnetism and the change in behavior of hysteresis curve by changing the magnetic doping

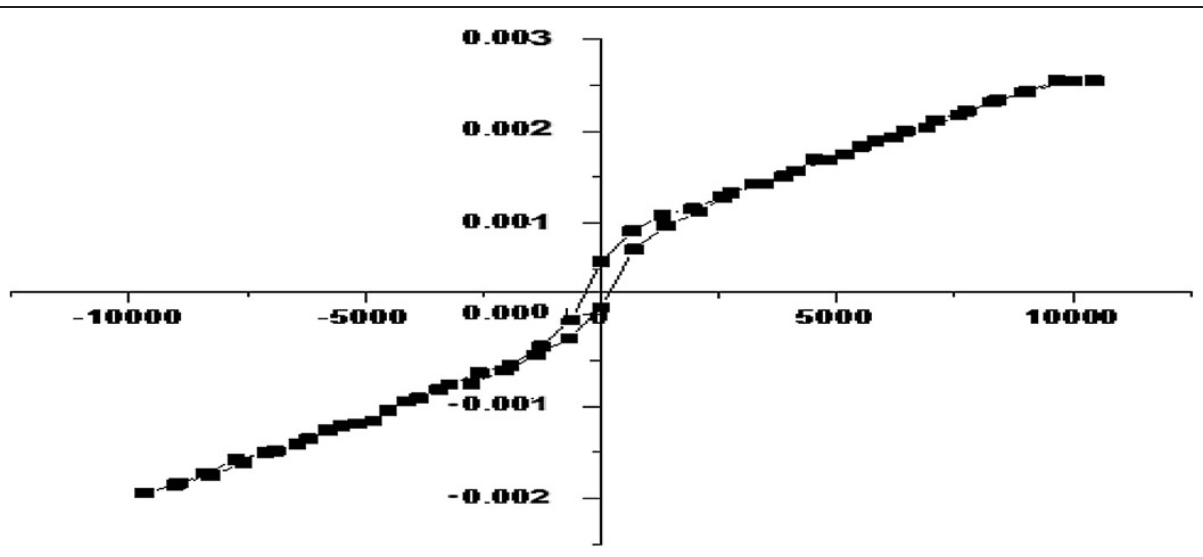

Figure 8 VSM profile of magnetic moment (emu) versus applied field $(G)$ curve of $3 \%$ cobalt-doped ZnO. 


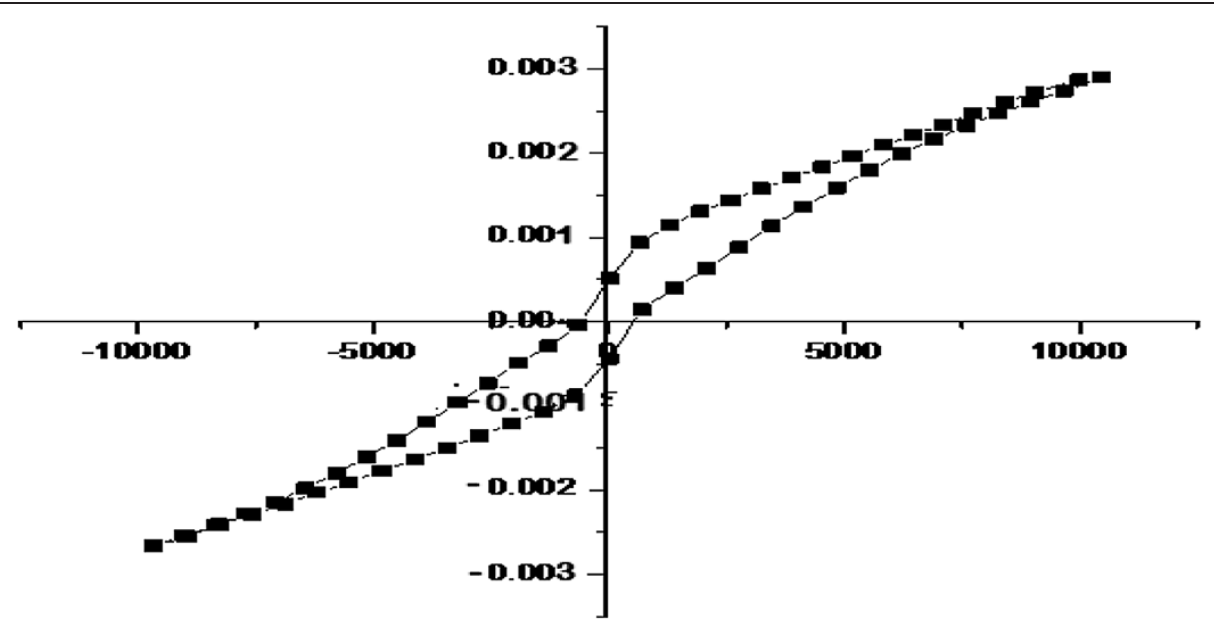

Figure 9 VSM profile of magnetic moment (emu) versus applied field (G) curve of $5 \%$ cobalt-doped ZnO.

concentration. The given Figures 6, 7, and 8 represent VSM profiles of Co-doped $\mathrm{ZnO}$ sketched at room temperature. Obtained profile confirms the presence of ferromagnetic behavior at room temperature and hence a successful doping where the impurity magnetic atom has successfully replaced the atoms of host crystal lattice, as XRD revealed pure $\mathrm{ZnO}$ at lower ratios. Presence of magnetic behavior depicts the presence of magnetic atoms. The smaller width of loop for $1 \%$ magnetic doping shows that it is a soft magnet, and 1\% doped sample does not contain any cluster of atoms or segregation. So, we may deduce that presence of ferromagnetic behavior is due to intrinsic coupling between the atoms of doped materials but not due to the presence of secondary phase (segregation). We observed a proportional change in the shape and width of hysteresis loop, a stronger loop for 3\% and 5\%, as shown in Figures 8 and 9, doped samples. These results were again contrasting, where some researchers observed ferromagnetic ordering at lower doping concentration but paramagnetic behavior for higher doping concentration. For iron doping, it was explained that paramagnetic behavior is due to antiferromagnetic coupling at higher doping concentration $[11,18-20]$. In our cobalt-doped samples, a cobalt proportional ferromagnetic strength was observed due to different electronic configurations of cobalt and agglomeration of a little fraction of cobalt atoms (as revealed by XRD) which prevents anti-ferromagnetic coupling.

\section{Conclusion}

Different compositions of cobalt-doped $\mathrm{ZnO}$ nanocrystallites were successfully fabricated using co-precipitation method. For fabrication of Co-doped $\mathrm{ZnO}$, decomposition temperate of precursor was determined by DSC/TGA analysis. XRD revealed standard $\mathrm{ZnO}$ profile for lower doping but additional phases were present for higher doping. Similarly FTIR and VSM studies also revealed doping proportional properties.

Competing interest

The authors declare that they have no competing interests.

\section{Authors' contributions}

MAS is the prime investigator, performer, analyzer, and writer of the study. SAS performed the data analysis and proofreading, and to whom correspondence is addressed. MN has done the PH and atmosphere control. KR performed the FTIR analysis. RA provided the technical guidance. All authors read and approved the final manuscript.

\section{Authors' information}

All authors are experienced researchers of Materials Science and Nanotechnology. SAS and RA are PhD faculty members; the others are senior PhD students.

\section{Acknowledgments}

Authors would like to acknowledge the chairman of the Centre for Advanced Study in Physics, GC University Lahore, for providing funds, access to $\mathrm{XRD}$, and for granting resources for VSM studies.

\section{Author details}

${ }^{1}$ Department of Physics, GC University, Lahore 54000, Pakistan. ${ }^{2}$ Department of Physics, Forman Christian College (University), Lahore 54000, Pakistan. ${ }^{3}$ Pakistan Council of Scientific and Industrial Research (PCSIR), Lahore 54000, Pakistan.

Received: 8 October 2012 Accepted: 8 October 2012 Published: 29 October 2012

\section{References}

1. Bunn, CW: The lattice dimensions of zinc oxide. Proc. Phys. Soc. 47, 836 (1935)

2. Damen, TC, Porto, SPS, Tell, B: Raman effect in zinc oxide. Phys. Rev. 142, 570 (1966)

3. Decremps, F, Pellicer-Porres, J, Saitta, AM, Chervin, JC, Polian, A: High-pressure Raman spectroscopy study of wurtzite ZnO. Phys. Rev. B. 65 092101 (2002)

4. Özgür, U, Alivov, Yl, Liu, C, Teke, A, Reshchikov, MA, Doğan, S, Vrutin, V, Cho, SJ, Morkoç, $\mathrm{H}$ : A comprehensive review of $\mathrm{ZnO}$ materials and devices. J. Appl. Phys. 98, 041301 (2003) 
5. Dietl, T, Ohno, H, Matsukura, F, Cibert, J, Ferrand, D: Zener model description of ferromagnetism in zinc-blende magnetic semiconductors. Science 287, 1019 (2000)

6. Ohno, Y, Young, DK, Beshoten, B, Matsukura, F, Ohno, H, Awschallon, DD: Electrical spin injection in a ferromagnetic semiconductor heterostructure. Nature 402, 790 (1999)

7. Pearton, SJ, Abernathy, CR, Overberg, ME, Thaler, GT, Nortan, DP, Theodoropoulou, NA, Hebard, AF, Park, YD, Ren, F, Kim, J, Boatner, LA: Magnetization and structural studies of $\mathrm{Mn}$-doped $\mathrm{ZnO}$ nanoparticles: prepared by reverse micelle method. J. Appl. Phys. 93, 1 (2003)

8. Wang, J: Ultrafine $\mathrm{ZnO}$ powder prepared by precipitation/mechanical milling. J. Mater. Sci. 36, 3273-3276 (2001)

9. Martiner, B, Sandiumenge, F, Fontcuberta, J: Ferromagnetism in Co-doped $\mathrm{ZnO}$ particles prepared by vaporization-condensation in a solar image furnace. J. Magn. Magn. Mater. 290, 168-170 (2004)

10. Wang, Z, Zhang, H, Zhang, L, Yuan, J, Yan, S, Wang, C: Low temperature synthesis of $\mathrm{ZnO}$ nanoparticles by solid state pyrolytic reaction. Nanotechnology 14, 11-15 (2003)

11. Caffarena, VD, Capitaneo, JL: Preparation of electrodeposited cobalt nanowires. Mat. Res. 9(2), 205-208 (2006)

12. Xue, DB, Chen, JS, Zhou, TJ, Chow, GM: Effect of Mn-doping on temperature dependent magnetic properties of $\mathrm{L} 1_{0} \mathrm{FeMnPt}$. J. Appl. Phys. 109, $07 B 747$ (2011)

13. Yang, $\mathrm{H}, \mathrm{Nie}$, S: Preparation and characterization of Co-doped ZnO nanomaterials. Mater. Chem. Phys. 114, 279-282 (2009)

14. Jayakumar, OD, Gopalakrishnan, IK: Surfactant induced enhanced room temperature ferromagnetism in $\mathrm{Zn}_{0.96} \mathrm{Mn}_{0.03} \mathrm{Li}_{0.01} \mathrm{O}$ nanoparticles prepared by solid state pyrolytic reaction. J. Cryst. Growth 307, 315-320 (2007)

15. Kim, HI, Choi, JM, Kim, DJ, So, MG: Synthesis and crystallization of fine SiC-Si3N4 composite powders by a vapor phase reaction. J. Ceram. Proc. Res. 3(3), 146-149 (2002)

16. Sui, XM, Shao, CL, Liu, YC: White-light emission of polyvinyl alcohol/ZnO hybrid nanofibers prepared by electrospinning. Appl. Phys. Lett. 87, 113-115 (2005)

17. Byrappa, K, Subramani, AK, Ananda, S, Lokanatha Rai, KM, Sunitha, MH Basavalingu, B, Soga, K: Impregnation of ZnO onto activated carbon under hydrothermal conditions and its photocatalytic properties. J. Mat. Sci. 41, 1355-1362 (2006)

18. Sharma, PK, Ranuk, DK, Avinash, PC: Effect of iron doping concentration on magnetic properties of $\mathrm{ZnO}$ nanoparticles. J. Magn. Magn. Mat. 321, 2587-2591 (2009)

19. Ghule, K, Ghule, AV, Chen, BJ, Ling, YC: Preparation and characterization of $\mathrm{ZnO}$ nanoparticles coated paper and its antibacterial activity study. Green Chem. 8, 1034-1041 (2006)

20. Ding, HM, Ding, J, Shi, Y, Liu, XY, Wang, J: Ultrafine ZnO powder prepared by precipitation/mechanical milling. J. Mat. Sci. 36, 3273-3276 (2001)

doi:10.1186/2228-5326-2-31

Cite this article as: Shafique et al:: Effect of doping concentration on absorbance, structural, and magnetic properties of cobalt-doped $\mathrm{ZnO}$ nano-crystallites. International Nano Letters 2012 2:31.

\section{Submit your manuscript to a SpringerOpen ${ }^{\circ}$ journal and benefit from:}

- Convenient online submission

- Rigorous peer review

- Immediate publication on acceptance

- Open access: articles freely available online

- High visibility within the field

- Retaining the copyright to your article

Submit your next manuscript at $>$ springeropen.com 